\title{
The Role of Stem Cells in Wound Angiogenesis
}

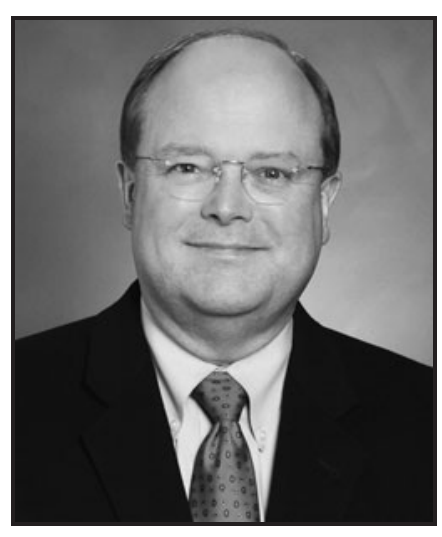

Timothy M. Crombleholme, MD

Submitted for publication July 17, 2013 Accepted in revised form March 19, 2014.

†Correspondence: Center for Children's Surgery, Division of Pediatric General, Thoracic and Fetal Surgery, Children's Hospital Colorado, School of Medicine, University of Colorado, 13123 East 16th Ave., Aurora, CO 80045 (e-mail: timothy.crombleholme@childrenscolorado.org).

\author{
Alice King, ${ }^{1, *}$ Swathi Balaji,,* Sundeep G. Keswani, \\ and Timothy M. Crombleholme $e^{2, \dagger}$ \\ 'Laboratory for Regenerative Wound Healing, Division of Pediatric, General, Thoracic and Fetal Surgery, Cincinnati \\ Children's Hospital, College of Medicine, University of Cincinnati, Cincinnati, Ohio. \\ ${ }^{2}$ Center for Children's Surgery, Division of Pediatric General, Thoracic and Fetal Surgery, Children's Hospital Colorado, \\ School of Medicine, University of Colorado, Aurora, Colorado.
}

Significance: Revascularization plays a critical role in wound healing and is regulated by a complex milieu of growth factors and cytokines. Deficiencies in revascularization contribute to the development of chronic nonhealing wounds.

Recent Advances: Stem-cell-based therapy provides a novel strategy to enhance angiogenesis and improve wound healing. With bioethical concerns associated with embryonic stem cells, focus has shifted to different populations of vascular precursors, isolated from adult somatic tissue. Three main populations have been identified: endothelial progenitor cells, mesenchymal stem cells, and induced-pluripotent stem cells. These populations demonstrate great promise to positively influence neovascularization and wound repair.

Critical Issues: Further studies to more definitively define each population are necessary to efficiently translate stem-cell-based therapeutic angiogenesis to the bedside. Better understanding of the physiologic pathways of how stem cells contribute to angiogenesis in normal tissue repair will help identify targets for successful therapeutic angiogenesis.

Future Directions: Active studies in both animal models and clinical trials are being conducted to develop effective delivery routes, including dosing, route, and timing. Stem-cell-based therapy holds significant potential as a strategy for therapeutic angiogenesis in the care of patients with chronic nonhealing wounds.

\section{SCOPE AND SIGNIFICANCE}

NeOvascularization Plays an essential role in wound healing and is regulated by a complex interaction between several growth factors, chemokines, and different cell populations. Stem cells have been shown to play an important role both through direct differentiation and incorporation as mature endothelial cells, as well as, a potent source of proangiogenic growth factors that support neovessel formation. With bioethical concerns and limitations of embry- onic stem cell (ES) research currently in place, this review provides an overview of three main populations of endothelial progenitors derived from adult tissue that demonstrate potential in augmenting vasculogenesis in wound healing, including endothelial progenitor cells (EPCs), mesenchymal stem cell (MSCs), and induced-pluripotent stem (iPS) cells.

\section{TRANSLATIONAL RELEVANCE}

Several groups have demonstrated that these vascular progeni-

*These authors contributed equally to this work. 
tor cells are capable of differentiating to endothelial cells, as well as the surrounding mesenchymal support cells required for vascular stability. Additionally, they have been shown to facilitate angiogenesis and improve wound healing through potent paracrine signaling, with production of a multitude of proangiogenic factors. Further research is necessary to refine isolation protocols and develop potential application modalities to bring stem-cell-based therapeutic angiogenesis to the bedside.

\section{CLINICAL RELEVANCE}

Each year, over $\$ 25$ billion dollars in U.S. healthcare is spent on chronic nonhealing wounds. A neovascularization deficit has been demonstrated in various disease states, such as diabetes and peripheral vascular diseases, which contributes strongly to impaired wound healing. Stemcell-based therapy provides a novel strategy to correct the angiogenic deficit by providing both structural components and proangiogenic growth factors.

\section{BACKGROUND}

Wound healing occurs in a highly coordinated sequence of overlapping but distinct phases in response to tissue injury: hemostasis, inflammation, proliferation, and maturation. Each phase represents a complex milieu of growth factors, cytokines, and different cell types resulting in coordinated cell migration, proliferation, neovascularization, extracellular matrix deposition, and maturation. ${ }^{1}$ Disruption of this complex process can result in chronic nonhealing wounds. The care of these chronic wounds represents a significant healthcare burden at $\$ 25$ billion per year in the United States ${ }^{2}$ and chronic wounds are found in patients with comorbidities, such as diabetes and peripheral vascular diseases. ${ }^{2}$ While the underlying pathogenesis of these nonhealing wounds is a multifactorial process, impaired neovascularization has been shown to be a common deficit in these pathologic wounds.

Neovascularization is necessary during all the phases of wound healing in order to provide nutrient delivery to the multitude of cells that regulate wound healing. The formation of new blood vessels occurs primarily through two independent processes: angiogenesis and vasculogenesis. Angiogenesis results from the sprouting of new capillaries from existing vessels. ${ }^{3}$ This occurs through endothelial cell activation, proliferation, and migration to form new vessels. ${ }^{4}$ Vasculogenesis describes de novo generation of vessels, a process dependent on bone-marrow-derived and circulating EPCs. In embryonic development, the primary precursor cells are termed angioblasts and hemangioblasts, which migrate, differentiate to endothelial cells, and then coalesce to form the vascular plexus and de novo vasculature. Postnatal vasculogenesis is mediated by progenitors to endothelial cells (Fig. 1). This rare cell population is being actively studied for both proangiogenic therapy to improve tissue repair and antiangiogenic therapy for cancer treatment. The premise of using stem cells to accelerate angiogenesis has been an active area of investigation. There is proof of concept for this strategy that was reported by several groups a decade ago, in which unprocessed bone marrow mononuclear cells (MNCs) were placed into sites of ischemia and demonstrated significant new blood vessel formation. ${ }^{5,6}$ These data suggest that there is a bone-marrow-derived cell that could directly contribute to neovascularization. Along with EPCs, various other populations of stem cells have been identified as possible targets for therapeutic angiogenesis and tissue repair.

Stem cells are classically defined as cells that have (1) the capacity to differentiate into specialized cell types and (2) the ability to self-renew, by dividing while maintaining an undifferentiated state. While ESs are pluripotent with an intrinsic ability to differentiate into all three germ layers, there are bioethical challenges in human ES research, from the production of new ES lines to the use of ES-based therapeutics in clinical trials. This review focuses on adult stem cells, which avoid the controversy of ESs and are derived without the utilization of embryos. Adult stem cells occur in smaller numbers and are commonly lineage restricted. Despite these limitations, adult stem cell research is an active field of research. Three main populations of stem cells have demonstrated potential in augmenting vasculogenesis in wound healing, including EPCs, MSCs, and iPS cells.

\section{EPCs: Defining the population}

EPCs are rare population of adult stem cells located within the stem cell niche of bone marrow. A small population of these immature hematopoietic endothelial cells is also found circulating in peripheral blood. First described in 1997 by Asahara et al., EPCs have since been isolated using a heterogeneous array of markers. ${ }^{7,8}$ Several studies have found significant heterogeneity among EPC populations in in vitro cultures. At least three types 


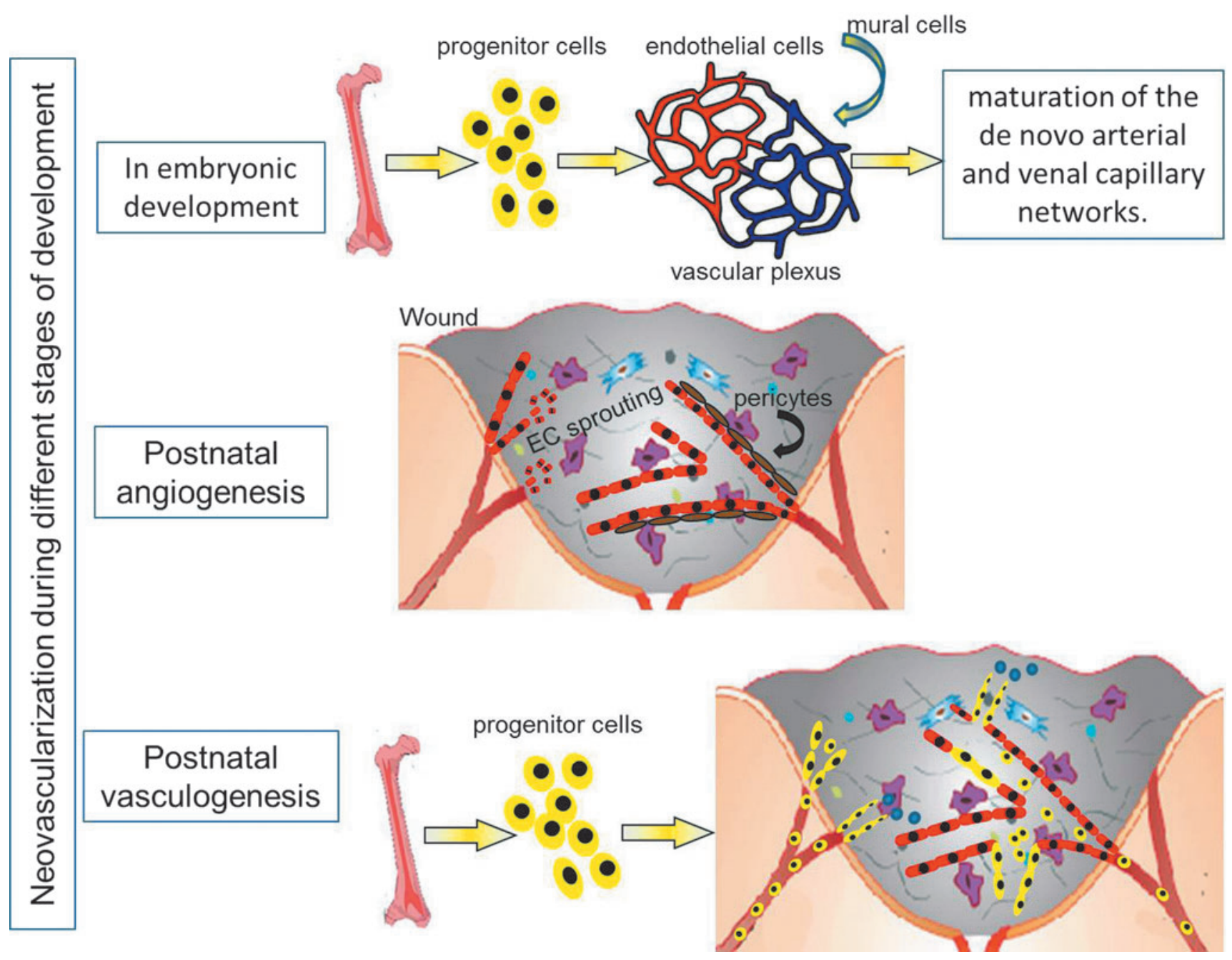

Figure 1. Neovascularization during different stages of development. In embryonic development, the primary precursor cells are termed angioblasts and hemangioblasts that migrate, differentiate to endothelial cells, and then coalesce to form the vascular plexus and de novo vasculature. In the postnatal setting, the formation of new blood vessels occurs primarily through two independent processes: angiogenesis and vasculogenesis. Angiogenesis results from the sprouting of new capillaries from existing vessels. This occurs through endothelial cell (EC) activation, proliferation, and migration to form new vessels that are then stabilized by pericytes. Vasculogenesis describes de novo generation of vessels, a process dependent on bone-marrow-derived and circulating endothelial progenitor cells (EPCs), which differentiate to endothelial cells and result in formation of new vessels. The differentiated endothelial cells also participate in reendothelialization and vessel repair. To see this illustration in color, the reader is referred to the web version of this article at www.liebertpub.com/wound

of EPCs have been described. Peripheral blood mononuclear cells contain cells termed "early EPCs" that share some endothelial but also monocytic characteristics and a restricted capacity for expansion. ${ }^{9}$ Recent studies have also shown the existence of a more promising population that originates from BM, circulates in peripheral blood, and whose morphology and proliferation pattern differs from the EPCs reported by Asahara et al. So-called endothelial outgrowth cells appear after 2 to 3 weeks of culture, rapidly replicate from multiple cells, and form into monolayers with a cobblestone-like morphology and a high proliferation capacity. ${ }^{10,11}$ Current data suggest that this population of outgrowing cells is a subset of true
EPCs deriving from BM that exhibit the potential for vascular repair after injury. More recently, Jung et al. also isolated and cultured two types of outgrowing cells obtained from the peripheral blood of patients with acute stroke, which were named endothelial or neuronal outgrowth cells, according to their morphological characteristics and protein or gene expression profiles. ${ }^{10}$ Both types of outgrowing cells maintained their proliferative capacities during a culture period of 3 months. Although numerous reports have described the clinical significance of circulating EPCs, there are few data that support their stem or progenitor status, namely, the ability to give rise to proliferating, functional endothelial cells such as 
outgrowing cells. EPCs have the ability to differentiate into endothelial cells. These cells are highly plastic and, depending on the microenvironment and presence of other cells, they can transdifferentiate and/or undergo cell fusion and become cells of different lineage, including cardiomyocytes, hepatocytes, and neurons, depending on the microenvironmental cues. EPCs have been shown to contribute to neovascularization in several models of tissue repair, including wound healing, retinopathy, myocardial ischemia, and peripheral vascular diseases. EPCs have also been suggested to play a critical role in tumor growth and metastasis (reviewed by Urbich and Dimmeler ${ }^{12}$ ).

No specific surface marker to definitively define the population has been identified. ${ }^{12}$ Currently, EPCs are being studied as part of cell populations isolated expressing a combination of hematopoietic lineage stem cell markers and markers of endothelial commitment. Similar to any other population of stem cells, the markers for EPCs continue to evolve. Multiple markers are used to define the population. The most common markers are CD34, FLK-1, CD133, and sca-1 along with functional assays, including their morphology and the ability to form colonies. ${ }^{13,14}$ Markers such as CD31, von Willebrand factor, and VE-cadherin that identify cells in a more advanced stage of endothelial maturation are also being used to identify the endothelial commitment. ${ }^{15}$

\section{EPCs in wound healing}

The vulnerary effects of EPCs are guided by signals initiated by endogenous stimuli, such as peripheral tissue hypoxia in wound healing. Wounding results in activation of the EPC cascade that includes (1) mobilization, (2) homing, (3) invasion, and (4) recruitment to the wound. The effect of EPCs on enhancing neovascularization is facilitated by differentiation into mature endothelial cells or paracrine signaling that enhances angiogenesis.

EPCs reside within the bone marrow niche and are subjected to low oxygen tension and high levels of stromal cell derived factor-1 (SDF-1). Peripheral tissue hypoxia following tissue trauma is a critical stimulus for tissue repair and is mediated by hypoxia inducible factor-1 alpha (HIF-1 $\alpha) .{ }^{16-18}$ Local hypoxia and increase in $\mathrm{HIF}-1 \alpha$ results in increased concentrations of EPC mobilizing factors, including vascular endothelial growth factor (VEGF), granulocyte-macrophage colony stimulating factor (GM-CSF), basic fibroblast growth factor (bFGF), placental growth factor, erythropoietin, and SDF-1. This results in an upregulation of protein- ases, such as matrix metalloproteinase-9 (MMP-9), which facilitates the release of the chemokine stem cell factor (SCF; soluble kit ligand). ${ }^{16,19}$ This MMP9-mediated increase in SCF results in mobilization of EPCs.

EPCs home toward the wound, following the gradient of cytokines and chemokines, such as VEGF and SDF-1. ${ }^{20}$ Increased levels of VEGF in the wound, either as a direct consequence of wounding or as following exogenous delivery using VEGFload pellets or Matrigel plug, result in increased recruitment of EPCs, with increased neovessel formation. Similarly, the interaction between increased SDF-1 expression present in hypoxic tissue and the highly expressed chemokine receptor CXCR-4 has been suggested to be a critical mediator in recruiting circulating EPCs.

Once activated and recruited to the wound bed, EPCs migrate through the blood vessel basement membrane to the interstitial extracellular matrix through an integrin-mediated process. ${ }^{21}$ The adhesion of EPCs in peripheral blood to activated endothelial sites in the wound bed is mediated primarily through the $\beta 2$-integrin subunit. ${ }^{22,23}$

Lastly, once the EPCs have arrived in the injured tissue, there is a recently reported novel strategy to maintain their levels in the tissue. Krishnamurthy et al. have used interleukin-10 to attenuate the inflammatory wound response, which results in increased number of EPCs that remain in the wound and enhanced neovascularization. ${ }^{24}$ This strategy to regulate inflammation in wounds may be an adjunct to other mechanisms employed to increase the number of EPCs and enhance their function.

\section{Clinical pathology resulting from EPC deficiency}

Although the population is rare and accounts for only about $0.0001 \%$ of total MNCs of peripheral blood in human adults, ${ }^{25}$ EPCs appear to have great clinical significance, likely in maintaining endothelial integrity and facilitating successful neovascularization following injury. ${ }^{26} \mathrm{EPC}$ dysfunction or deficiency has been implicated in multiple vascular pathologies, including peripheral ischemia, myocardial ischemia following myocardial infarction, and diabetes. Multiple comorbidities and risk factors associated with impaired or delayed wound healing have demonstrated EPC deficiencies. ${ }^{16}$ For example, smoking has been associated with reduced numbers of circulating EPCs; hypertension is associated with delayed wound healing, which is associated with impaired migration of EPCs; and diabetes has been associ- 
ated with reduced EPCs and impaired potential to augment angiogenesis. ${ }^{16,27-30}$

\section{Therapeutic potential of EPCs}

While translation of EPCs specifically to cutaneous wound healing in clinical studies is pending, studies in other models of tissue repair have demonstrated promising improvement following transplantation of EPCs. ${ }^{31,32}$ In patients with peripheral arterial disease resulting in lower extremity ischemia, transplantation of bone marrow MNCs into the gastrocnemius compared with transplantation of peripheral blood MNCs resulted in clinical improvement with decreased rest pain, and increased pain-free walk time with improved ankle-brachial index and improved transcutaneous oxygen pressure. ${ }^{5}$ In patients with re-perfused acute myocardial infarction, transplantation of autologous EPCs via an intracoronary infusion resulted in improved left ventricular ejection fraction, reduced pathologic remodeling, and increased myocardial viability in the infarct zone. ${ }^{33}$ In this study, improvements were noted with both bonemarrow- and peripheral-blood-derived EPCs. ${ }^{33}$ Strauer et al. demonstrated similar improvements in acute myocardial infarction patients with improved stroke volume, contractility, decrease in infarct region size, and perfusion of the infarction area 3 months following transplant of autologous bone marrow to the affected coronary. ${ }^{34}$

Novel strategies beyond autologous transplantation may lie in augmenting EPC recruitment (Fig. 2). We have shown that the injection of SCF is sufficient to mobilize stem cells after pneumonectomy (unpublished data). Another recent study by our group in a murine wounding model has suggested that intravenous injection of SCF results in increased EPC numbers in peripheral blood as well as within the wound bed (unpublished data). However, there was also an associated increase in inflammatory response in the wound bed. SCFinduced EPC recruitment in conjunction with the addition of anti-inflammatory mediators, such as interleukin-10, may prove to be a viable therapeutic strategy. Localized increase of VEGF through implantation of VEGF pellets or VEGFloaded Matrigel plugs is capable of stimulating neovascularization with a large contribution from EPCs. Galiano et al. demonstrated that topical VEGF can improve diabetic wound healing by locally upregulating growth factors, such as PDGF-B and FGF, that are important for tissue repair and by systemically mobilizing and recruiting bonemarrow-derived EPCs to the local wound environ-

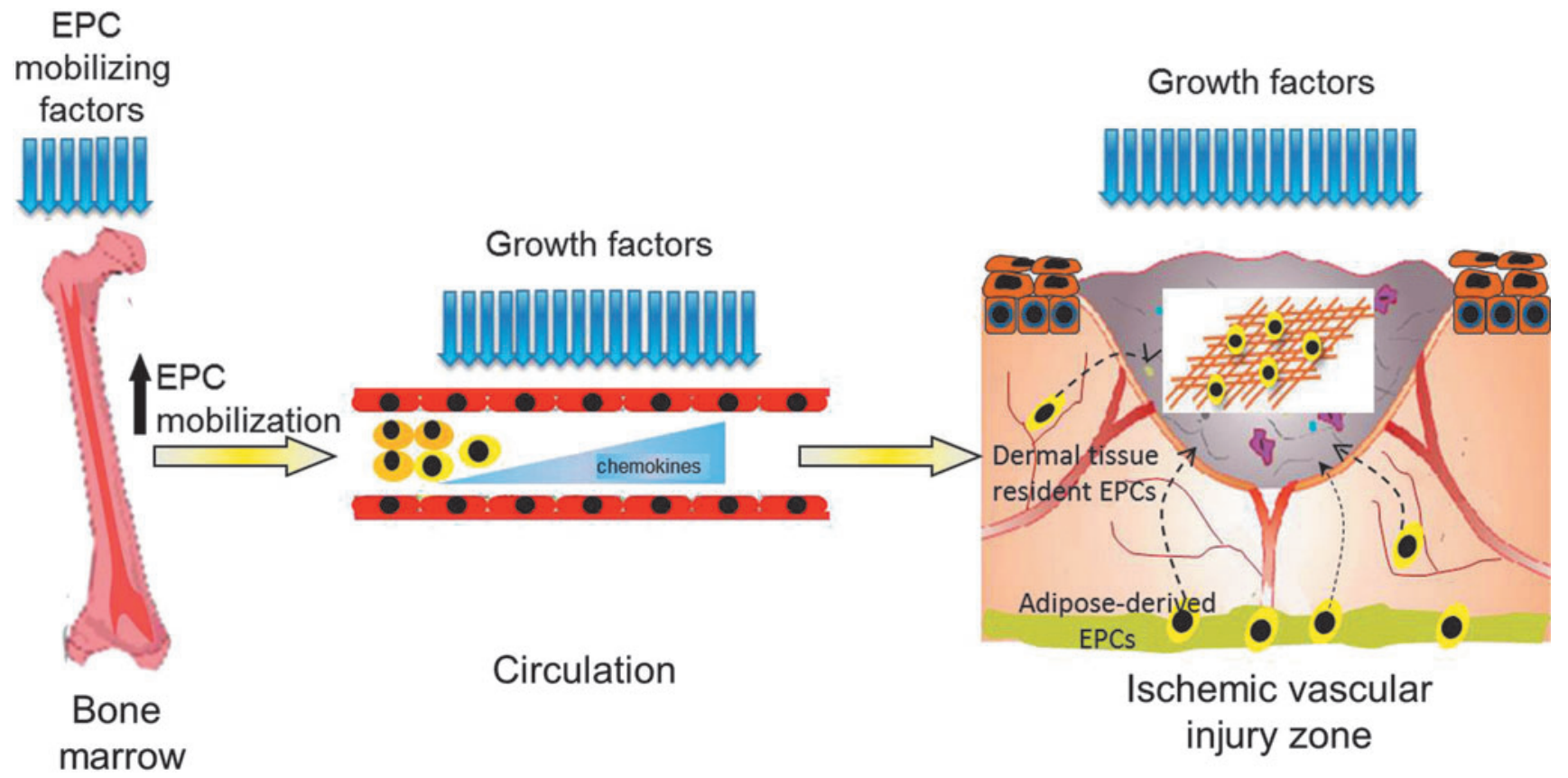

Figure 2. Novel strategies beyond autologous transplantation may lie in augmenting EPC recruitment. Injection of EPC mobilizing factors, such as stem cell factor (SCF) or granulocyte colony stimulation factors, can increase mobilization of stem cells from bone marrow. Growth factor supplementation to modify EPC adhesion can also increase EPC-mediated neovascularization. Additionally, localized increase of growth factors such as VEGF, through implantation of VEGF pellets or VEGF-loaded matrigel plugs, is capable of stimulating neovascularization with a large contribution from EPCs. VEGF, vascular endothelial growth factor. To see this illustration in color, the reader is referred to the web version of this article at www.liebertpub.com/wound 
ment where they are able to accelerate repair. ${ }^{35}$ Additionally, efforts to encode EPCs with VEGF through gene transfer ${ }^{36,37}$ have also demonstrated increased revascularization in a hind limb ischemia model, compared with nontransduced EPC injection. Modification of EPC adhesion also represents an alternative therapeutic strategy. ${ }^{36} \mathrm{Ex}-$ posure of human EPCs to simvastatin has been shown to upregulate integrins $\alpha_{5} \beta_{1}$ and $\alpha_{v} \beta_{5}$, both known to play a role in angiogenesis. In a rat model of balloon-injury-induced arterial injury, these simvastatin-treated EPCs demonstrated increased incorporation of endothelial cells. ${ }^{38}$ These alternative strategies represent different avenues of study that may be combined to maximize the therapeutic potential of EPC-mediated neovascularization in wound healing. ${ }^{39}$

Autologous EPCs have recently been used in tissue-engineered vascular grafts. Grafts seeded with autologous $\mathrm{CD}^{+} 4^{+}$cells from canine bone marrow and then implanted into the aorta were found to have increased surface endothelialization and vascularization compared with controls. ${ }^{40} E x$ vivo-expanded autologous EPC-seeded decellularized porcine iliac vessel grafts remained patent for 130 days as a carotid interposition graft in sheep, compared with nonseeded grafts that occluded within 15 days. ${ }^{41}$

Although the role of EPCs in neovascularization has been convincingly shown by several groups, a major therapeutic limitation lies in the large variation in findings between groups. For example, the rate of incorporation of EPCs into newly formed vessels has a wide range. The basal incorporation rate of progenitor cells without tissue injury is extremely low. ${ }^{42}$ In ischemic tissue, the incorporation rate of genetically labeled bone-marrow-derived cells, which coexpress endothelial marker proteins, differs from $0 \%$ to $90 \%$ incorporation. ${ }^{12,42-44}$ Likewise, the extent of incorporation of bone-marrowderived cells in cerebral vessels after stroke varies in the literature. Two studies reported positive vessels with an average of $34 \%$ endothelialmarker-expressing, bone-marrow-derived cells ${ }^{45}$; other groups could not detect endothelial-markerexpressing cells. ${ }^{46}$ High amounts $(>50 \%)$ were predominantly detected in models of tumor angiogenesis. ${ }^{47}$ Some studies only detected bonemarrow-derived cells adjacent to vessels, which do not express endothelial marker proteins. ${ }^{48,49}$ While part of this phenotypic heterogeneity likely lies in differences in the tissue and microenvironment of the wound (intensity of injury or ischemia), the field is challenged with the absence of standardized isolation protocols and definitive surface markers.
Future research to better define the population is needed to move forward with therapeutic interventions.

\section{MSCs: Defining the population}

MSCs are multipotent nonhematopoietic stromal cells capable of self-renewal. They have been isolated from bone marrow, as well as various other tissues, including adipose, gingiva, muscles, and the umbilical cord. Depending on the microenvironment, MSCs have been differentiated to various cell types, including bone, cartilage, muscle, fat, and endothelium ${ }^{50}$ (Fig. 3). Residing in perivascular locations, MSCs are thought to represent a subpopulation of pericytes, resident tissue cells that act as support cells for blood vessels and help to stabilize the endothelial network. While EPCs facilitate angiogenesis both by direct differentiation to endothelial cells and through paracrine actions, MSCs typically facilitate revascularization through differentiation into pericytes and paracrine secretion of growth factors. Recent studies have demonstrated the ability to differentiate MSCs to myofibroblasts, pericytes, as well as endothelial cells ${ }^{51}$; the clinical relevance of this in vitro differentiation remains unknown. MSCs readily respond to injury and inflammation following wounding. In particular, localized hypoxia mediates a favorable MSC response with enhanced self-renewal and increased release of proangiogenic factors, such as VEGF, bFGF, and angiopoietin (Ang-1). Additionally, MSCs contribute to wound healing by cellular differentiation for structural repair of wounds and immune regulation by reducing $\mathrm{T}$ cell proliferation.

\section{Therapeutic potential of MSCs}

MSCs have been demonstrated to enhance tissue repair in multiple models of injury, including skin, kidney, myocardium, lung, and brain. Diverse pathologies, such as cutaneous wounds, chronic renal failure ${ }^{52}$ acute kidney injury, ${ }^{53}$ renal fibrosis, ${ }^{54}$ acute myocardial infarction, ${ }^{55}$ asthma, ${ }^{56}$ and brain ischemia, ${ }^{57}$ demonstrated enhanced healing with attenuation of inflammation response and improved revascularization.

Multiple strategies are being explored in order to augment wound repair through application of MSCs. MSC transplantation results in relatively low rate of differentiation and engraftment as structural components, leading to theory that the vulnerary effects of MSCs are facilitated primarily through paracrine effects. This theory is supported by data in which MSC-conditioned media was administered to deep-burn wounds in rats and exci- 


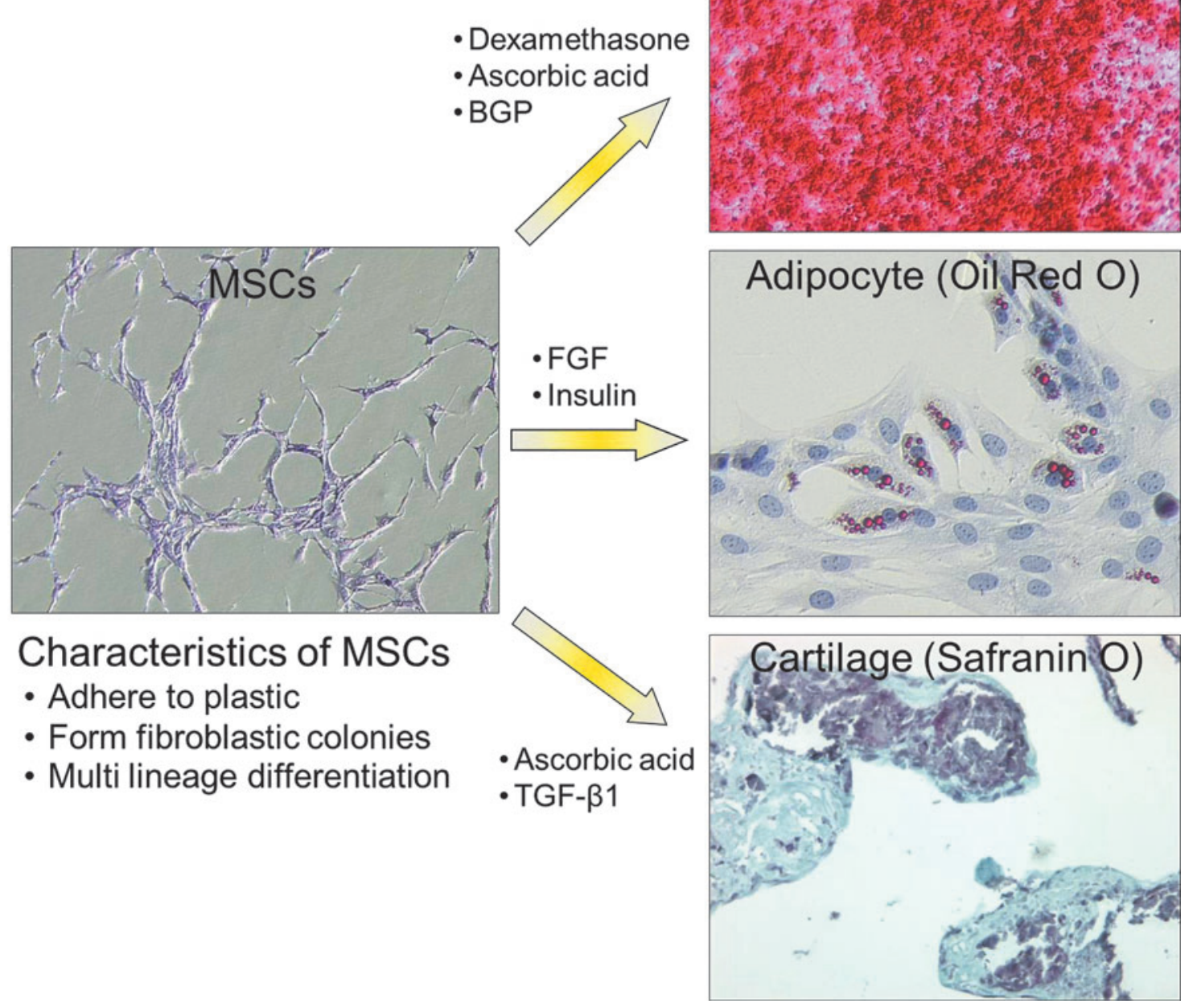

Figure 3. The mesengenic process. The three criteria accepted regarding mesenchymal stem cell (MSC) isolation from bone marrow are as follows: these cells adhere to plastic, they form fibroblast-like colonies, and they have the potential to differentiate into multiple lineages, including bone, muscle, or adipose tissue, depending on the stimuli to which they are exposed to in vitro. MSCs can expand for more than 50 passages and form fibroblast-like colonies that increase in number with more passages as more cell contaminants are selected out. To see this illustration in color, the reader is referred to the web version of this article at www.liebertpub.com/wound

sional wounds in mice. MSC-conditioned media enhanced wound healing and re-epithelialization with increased revascularization, granulation tissue formation, and decreased inflammatory cell infiltration. ${ }^{58}$ Intravenous injection of MSCs isolated from GFP transgenic mice to cutaneous wounds in control mice demonstrated that MSCs facilitate rapid wound repair and are capable of differentiating into keratinocytes, endothelial cells, and pericytes. ${ }^{59}$ Development of MSCs as cell sheets has demonstrated improved healing when applied topically to full-thickness murine wounds. ${ }^{60}$ More recently, the interactions between MSCs and EPCs have been studied. Rouwkema et al. demonstrated that coculture provoked angiogenic differentiation and tubulogenesis in cases were BM-EPCs were mature enough, but not in early BM-EPCs, concluding that the maturation stage of BM-EPCs was of critical importance in the final outcome of the coculture. ${ }^{61}$ Cotransplantation of MSCs and EPCs has been demonstrated to significantly improve diabetic wound healing ${ }^{62}$ and improve healing of complex bone defects. ${ }^{63}$

Clinical translation of the therapeutic use of MSCs in wound healing has continued with promising results. Various delivery methods are actively being explored in order to augment healing in both acute and chronic wounds. Auto- 


\section{Embryonic Stem cells}

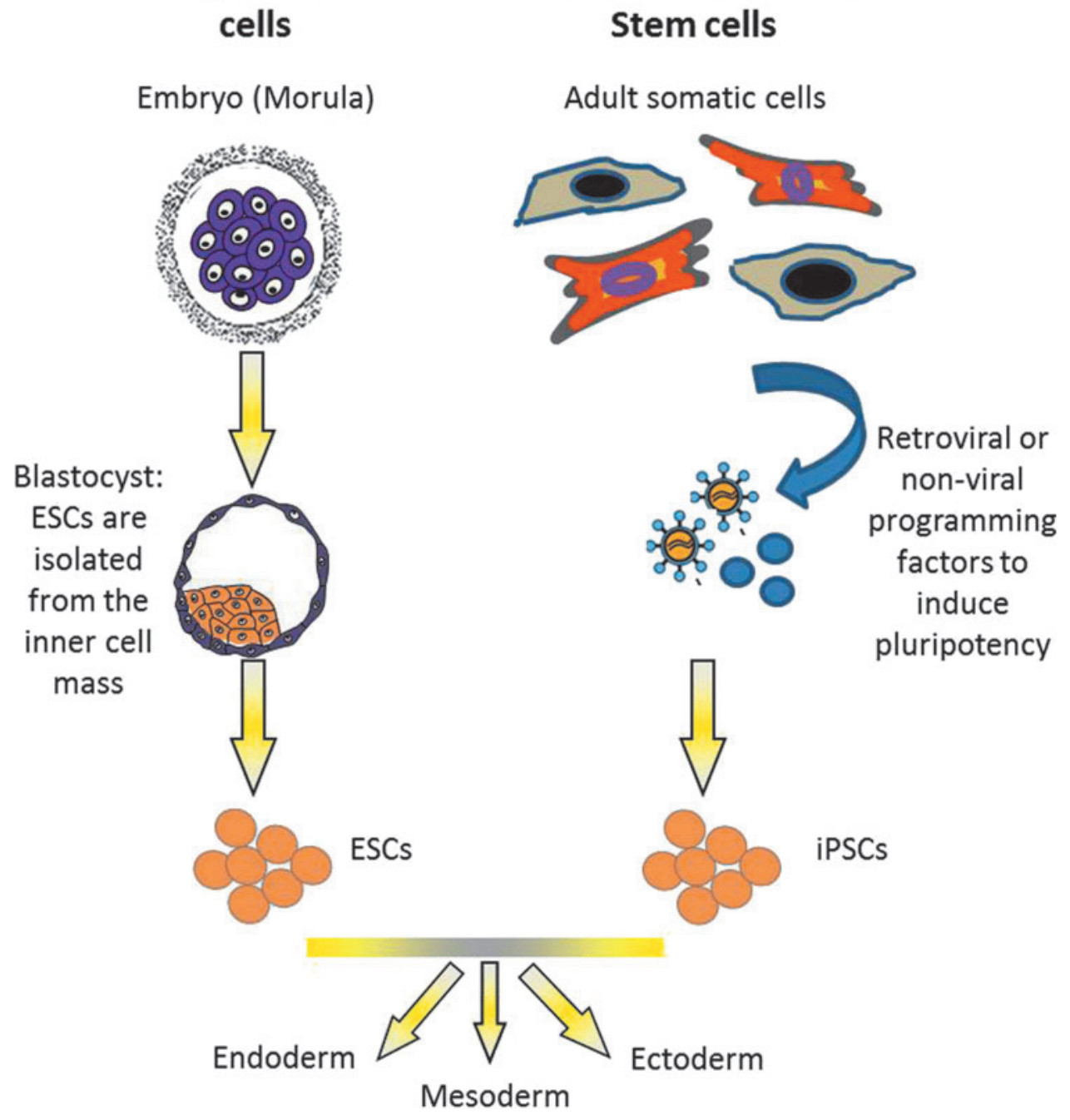

\section{Induced Pluripotent} Stem cells

Figure 4. While embryonic stem cells (ESs) are pluripotent with an intrinsic ability to differentiate into all three germ layers, there are bioethical challenges in human ES research, from the production of new ES lines to the use of ES-based therapeutics in clinical trials. Induced pluripotent stem cells have a similar ability to differentiate into all three germ layers; however, these are generated from adult somatic cells, enabling clinical translation of these cells. Differentiated adult cells, such as fibroblasts, can be reprogrammed to pluripotent states through exposure to a defined set of transcription factors, including octamer 3/4 (0ct3), sex-determining region Y-box (Sox2), Kruppel-like factor 4 (Klf4), and v-Myc myelocytomatosis viral oncogene homolog (c-Myc), using retroviral vectors or nonviral techniques. ESCs, embryonic stem cells; iPSCs, induced-pluripotent stem cells. To see this illustration in color, the reader is referred to the web version of this article at www.liebertpub.com/wound

logous transplantation of MSCs delivered using a fibrin spray to acute surgical wounds as well as chronic nonhealing wounds of lower extremities demonstrated accelerated wound closure in a dose-dependent fashion. ${ }^{50}$ Similarly, use of a collagen scaffold seeded with allogeneic nondiabetic MSCs to nonhealing diabetic foot ulcers resulted in increased angiogenesis and improved wound closure. ${ }^{64}$ An additional potential therapeutic method by which MSCs can be used to promote neovascularization is to isolate the cells from a patient, expand them on a large scale, and then bank them for when needed for autologous transplant. These MSCs can be modified by gene ma- nipulation techniques to support angiogenesis or other vulnerary functions.

Future directions that will further broaden therapeutic applicability will include the incorporation of MSCs into skin substitutes to augment healing with both paracrine signaling and effector cells, such as fibroblasts and keratinocytes.

The therapeutic potential of MSCs is incredibly promising with advantages including avoiding bioethical concerns associated with ESs, as well as the ability to avoid immunogenicity when isolated from the patient, differentiated to the desired cell type, and re-implanted. Also, unlike the relatively rare population of EPCs, MSCs can be obtained 
in large quantities with low risk to patients from multiple somatic tissue sources. MSCs demonstrate a large range of phenotypic heterogeneity, which may in large part be due to the lack of standardized isolation protocols. MSCs are harvested from multiple tissue sources through a variety of techniques. Further studies are necessary to determine whether the MSCs isolated from these different sources are truly comparable populations, or whether they need to be further subdivided and categorized. While a true limitation, this possible subclassification of MSCs may also potentially yield new therapeutic applications for a more homogenous population of cells.

\section{Induced pluripotent stem cells: Defining the population}

In 2006, Takahashi et al. ${ }^{65}$ developed iPS cells from postnatal somatic cells that are reprogrammed with ES-like characteristics. They are generated through exposure to a defined set of transcription factors using a retroviral vector, including octamer 3/4 (Oct3), sex-determining region Y-box (Sox2), Kruppel-like factor 4 (Klf4), and v-Myc myelocytomatosis viral oncogene homolog (c-Myc) (Fig. 4). Using this strategy, differentiated cells, such as fibroblasts, have been reprogrammed to pluripotent states. iPS cell technology can be used to model human disorders; create cell-based models of human diseases, including neurodegenerative diseases $^{66}$; and in drug discovery and establishing therapeutic strategies. iPS cells have been shown to differentiate to cardiac muscle, vascular smooth muscle, and pericytes. Although this field continues to be in its infancy, Niwa et al. have demonstrated that murine iPS cells generated from fibroblasts were able to differentiate into hematopoietic stem cells and endothelial cells through coculture with OP9 stromal cells. ${ }^{51}$

The therapeutic potential of iPS cells is promising for a multitude of disease processes, including augmentation of revascularization during wound healing. ${ }^{8}$ Therapeutic iPS cells would permit isolation of differentiated cells from a patient, reprogramming to a pluripotent state, followed by differentiation to the desired cell types for reimplantation. As autologous tissue, this strategy avoids issues of immunogenicity. Additionally, bioethical concerns for use of ESs are avoided, while achieving what appears to be similar pluripotency of ESs. As the field continues to advance, techniques to refine and increase the frequency of progenitor cell development are needed. Specifically for vascular regeneration, improved selection markers and optimized protocols will be needed to drive cells to an endothelial lineage. ${ }^{51}$ Similarly, before iPS cells progress to human trials, several issues will need to be addressed. This includes the need for viral vectors to reprogram the somatic cells, which incurs the potential for insertional mutagenesis with vectors such as retrovirus and lentiviruses. This may potentially be overcome by the use of adenoviral vectors and plasmids to manipulate cells. Additionally, several nonviral-mediated methods of reprogramming cells are underway. An additional issue that must be addressed is the relative inefficiency in the reprogramming process with the current time 
course taking 3 to 4 weeks that also yields a relatively low number of cells. As our understanding of the intricacies of reprogramming cells increases, the process will become more efficient. Lastly, one major potential issue is that the isolation of these cells from individual patients with chronic diseases may yield iPS cells predisposed to the pathologic defect that may not be as effective in therapeutic potential. Despite these concerns, iPS cells have the potential to have a large therapeutic impact in a variety of pathologic conditions.

\section{FUTURE DIRECTIONS}

There have been significant scientific advances in the field of stem-cell-based therapies for neovascularization. Further studies are required to more definitively define each population and to understand the heterogeneity within each group. ${ }^{67}$ Enhanced knowledge of the physiologic pathways of how stem cells contribute to angiogenesis in normal tissue repair will help identify targets for successful therapeutic angiogenesis. ${ }^{68,69}$ Direct autologous application of cells to a site of impaired neovascularization or novel strategies such as implantation of artificial grafts with pluripotent cells to augment reperfusion can promote neovascularization in impaired tissue. As our understanding of EPCs, MSCs, and iPS cells evolves, specific clinical indications will become apparent for each cell type.

\section{AUTHOR DISCLOSURE AND GHOSTWRITING}

There are no competing financial interests for A.K., S.B., S.G.K., and T.M.C. The content of this article is expressly written by the authors listed. No ghostwriters were used to write this article.

\section{ABOUT THE AUTHORS}

Alice King, MD, is a University of Cincinnati General Surgery resident who is completing a 2year postdoctoral research fellowship in the laboratory for regenerative wound healing at CCHMC. Swathi Balaji, PhD, received her $\mathrm{PhD}$ in Biomedical Engineering from University of Cincinnati. She is a postdoctoral research fellow in the laboratory for regenerative wound healing at CCHMC specializing in mechanisms underlying the fetal regenerative wound healing. Sundeep G. Keswani, MD, is a pediatric and fetal surgeon at CCHMC. He is the Principal Investigator of the laboratory for regenerative wound healing at CCHMC focused on elucidating the mechanisms underlying the fetal regenerative phenomenon, and is also the director of Pediatric Wound Care Center at CCHMC. Timothy M. Crombleholme, MD, is Surgeon-in-Chief at Children's Hospital, Colorado, and he had extensive NIH funding to study the role of EPCs in wound healing and neovascularization.

\section{REFERENCES}

1. Ko SH, Nauta A, Wong V, Glotzbach J, Gurtner $\mathrm{GC}$, and Longaker MT: The role of stem cells in cutaneous wound healing: what do we really know? Plast Reconstr Surg 2011; 127 Suppl 1: $10 \mathrm{~S}$.

2. Sen CK, Gordillo GM, Roy S, et al:: Human skin wounds: a major and snowballing threat to public health and the economy. Wound Repair Regen 2009; 17: 763

3. Isner JM and Asahara T: Angiogenesis and vasculogenesis as therapeutic strategies for postnatal neovascularization. J Clin Invest 1999; 103: 1231

4. Carmeliet P: Angiogenesis in life, disease and medicine. Nature 2005; 438: 932.

5. Tateishi-Yuyama E, Matsubara H, Murohara T, et al:: Therapeutic angiogenesis for patients with limb ischaemia by autologous transplantation of bone-marrow cells: a pilot study and a randomised controlled trial. Lancet 2002; 360: 427.
6. Badiavas EV and Falanga V: Treatment of chronic wounds with bone marrow-derived cells. Arch Dermatol 2003; 139: 510.

7. Asahara T, Murohara T, Sullivan A, et al.: Isolation of putative progenitor endothelial cells for angiogenesis. Science 1997; 275: 964.

8. Leeper NJ, Hunter AL, and Cooke JP: Stem cell therapy for vascular regeneration: adult, embryonic, and induced pluripotent stem cells. Circulation 2010; 122: 517.

9. Hur J, Yoon $\mathrm{CH}$, Kim HS, et al.: Characterization of two types of endothelial progenitor cells and their different contributions to neovasculogenesis. Arterioscler Thromb Vasc Biol 2004; 24: 288.

10. Jung KH, Chu K, Lee ST, et al.: Identification of neuronal outgrowth cells from peripheral blood of stroke patients. Ann Neurol 2008; 63: 312.

11. Lin Y, Weisdorf DJ, Solovey A, and Hebbel RP: Origins of circulating endothelial cells and endo- thelial outgrowth from blood. J Clin Invest 2000; 105: 71.

12. Urbich $\mathrm{C}$ and Dimmeler $\mathrm{S}$ : Endothelial progenitor cells: characterization and role in vascular biology. Circ Res 2004; 95: 343.

13. Tian F, Liang PH, and Li LY: Inhibition of endothelial progenitor cell differentiation by VEGI. Blood 2009; 113: 5352.

14. Schatteman GC, Dunnwald M, and Jiao C: Biology of bone marrow-derived endothelial cell precursors. Am J Physiol Heart Circ Physiol 2007; 292: H1.

15. Peichev M, Naiyer AJ, Pereira D, et al.: Expression of VEGFR-2 and AC133 by circulating human CD34 $(+)$ cells identifies a population of functional endothelial precursors. Blood 2000; 95: 952.

16. Goldstein LJ, Gallagher KA, Bauer SM, et al:: Endothelial progenitor cell release into circulation is triggered by hyperoxia-induced increases in 
bone marrow nitric oxide. Stem Cells 2006; 24: 2309 .

17. Aicher A, Heeschen C, Mildner-Rihm C, et al:: Essential role of endothelial nitric oxide synthase for mobilization of stem and progenitor cells. Nat Med 2003; 9: 1370

18. De Falco E, Porcelli D, Torella AR, et al:: SDF-1 involvement in endothelial phenotype and ischemia-induced recruitment of bone marrow progenitor cells. Blood 2004; 104: 3472

19. Dimmeler S, Fleming I, Fisslthaler B, Hermann C, Busse R, and Zeiher AM: Activation of nitric oxide synthase in endothelial cells by Akt-dependent phosphorylation. Nature 1999; 399: 601.

20. Caiado F, Real C, Carvalho T, and Dias S: Notch pathway modulation on bone marrow-derived vascular precursor cells regulates their angiogenic and wound healing potential. PLoS One 2008; 3: e3752.

21. Hynes R0: The extracellular matrix: not just pretty fibrils. Science 2009; 326: 1216.

22. Caiado F and Dias S: Endothelial progenitor cells and integrins: adhesive needs. Fibrogenesis Tissue Repair 2012; 5: 4

23. Chavakis E, Aicher A, Heeschen C, et al:: Role of beta2-integrins for homing and neovascularization capacity of endothelial progenitor cells. J Exp Med 2005; 201: 63

24. Krishnamurthy $P$, Thal M, Verma S, et al:: Interleukin-10 deficiency impairs bone marrow-derived endothelial progenitor cell survival and function in ischemic myocardium. Circ Res 2011; 109: 1280.

25. Ingram DA, Caplice NM, and Yoder MC. Unresolved questions, changing definitions, and novel paradigms for defining endothelial progenitor cells. Blood 2005; 106: 1525.

26. Rafii S, Heissig B, and Hattori K. Efficient mobilization and recruitment of marrow-derived endothelial and hematopoietic stem cells by adenoviral vectors expressing angiogenic factors. Gene Ther 2002; 9: 631 .

27. Loomans CJ, de Koning EJ, Staal FJ, et al:: Endothelial progenitor cell dysfunction: a novel concept in the pathogenesis of vascular complications of type 1 diabetes. Diabetes 2004; 53 195.

28. Suh W, Kim KL, Kim JM, et al:: Transplantation of endothelial progenitor cells accelerates derma wound healing with increased recruitment of monocytes/macrophages and neovascularization. Stem Cells 2005; 23: 1571.

29. Georgescu A, Alexandru N, Constantinescu A, Titorencu I, and Popov D. The promise of EPCbased therapies on vascular dysfunction in diabetes. Eur J Pharmacol 2011; 669: 1.

30. Tepper OM, Galiano RD, Capla JM, et al:: Human endothelial progenitor cells from type II diabetics exhibit impaired proliferation, adhesion, and incorporation into vascular structures. Circulation 2002: 106: 2781.
31. Kawamoto A, Gwon HC, Iwaguro H, et al:: Therapeutic potential of ex vivo expanded endothelial progenitor cells for myocardial ischemia. Circulation 2001; 103: 634

32. Madonna R and De Caterina R. Cellular and molecular mechanisms of vascular injury in diabetes-part II: cellular mechanisms and therapeutic targets. Vasc Pharmacol 2011; 54: 75.

33. Assmus B, Schachinger V, Teupe C, et al.: Transplantation of progenitor cells and regeneration enhancement in acute myocardial infarction (TOPCARE-AMI). Circulation 2002; 106: 3009.

34. Strauer BE, Brehm M, Zeus T, et al:: Repair of infarcted myocardium by autologous intracoronary mononuclear bone marrow cell transplantation in humans. Circulation 2002; 106: 1913.

35. Galiano RD, Tepper OM, Pelo CR, et al:: Topical vascular endothelial growth factor accelerates diabetic wound healing through increased angiogenesis and by mobilizing and recruiting bone marrow-derived cells. Am J Pathol 2004; 164: 1935.

36. Iwaguro H, Yamaguchi J, Kalka C, et al.: Endothelial progenitor cell vascular endothelial growth factor gene transfer for vascular regeneration. Circulation 2002; 105: 732.

37. Kalka C, Masuda H, Takahashi T, et al:: Vascular endothelial growth factor(165) gene transfer augments circulating endothelial progenitor cells in human subjects. Circ Res 2000; 86: 1198.

38. Walter DH, Rittig K, Bahlmann FH, et al:: Statin therapy accelerates reendothelialization: a novel effect involving mobilization and incorporation of bone marrow-derived endothelial progenitor cells. Circulation 2002; 105: 3017

39. Losordo DW and Dimmeler S. Therapeutic angiogenesis and vasculogenesis for ischemic disease: part II: cell-based therapies. Circulation 2004; 109: 2692.

40. Bhattacharya V, McSweeney PA, Shi 0 , et al.: Enhanced endothelialization and microvessel formation in polyester grafts seeded with CD34(+) bone marrow cells. Blood 2000; 95: 581.

41. Kaushal S, Amiel GE, Guleserian KJ, et al.: Functional small-diameter neovessels created using endothelial progenitor cells expanded ex vivo. Nat Med 2001; 7: 1035

42. Crosby JR, Kaminski WE, Schatteman G, et al:: Endothelial cells of hematopoietic origin make a significant contribution to adult blood vessel formation. Circ Res 2000; 87: 728.

43. Jackson KA, Majka SM, Wang $H$, et al.: Regeneration of ischemic cardiac muscle and vascular endothelium by adult stem cells. J Clin Invest 2001; 107: 1395

44. Lyden D, Hattori K, Dias S, et al:: Impaired recruitment of bone-marrow-derived endothelial and hematopoietic precursor cells blocks tumor angiogenesis and growth. Nat Med 2001; 7: 1194
45. Hess DC, Hill WD, Martin-Studdard A, Carroll J Brailer $\mathrm{J}$, and Carothers $\mathrm{J}$ : Bone marrow as a source of endothelial cells and NeuN-expressing cells after stroke. Stroke 2002; 33: 1362

46. Machein MR, Renninger S, de Lima-Hahn E, and Plate $\mathrm{KH}$ : Minor contribution of bone marrowderived endothelial progenitors to the vascularization of murine gliomas. Brain Pathol 2003; 13: 582.

47. Garcia-Barros M, Paris F, Cordon-Cardo C, et al.: Tumor response to radiotherapy regulated by endothelial cell apoptosis. Science 2003; 300: 1155

48. De Palma M, Venneri MA, Roca C, and Naldini L: Targeting exogenous genes to tumor angiogenesis by transplantation of genetically modified hematopoietic stem cells. Nat Med 2003; 9: 789.

49. Ziegelhoeffer T, Fernandez B, Kostin S, et al:: Bone marrow-derived cells do not incorporate into the adult growing vasculature. Circ Res 2004; 94: 230 .

50. Falanga V, Iwamoto S, Chartier M, et al:: Autologous bone marrow-derived cultured mesenchymal stem cells delivered in a fibrin spray accelerate healing in murine and human cutaneous wounds. Tissue Eng 2007; 13: 1299

51. Niwa A, Umeda $K$, Chang $H$, et al.: Orderly hematopoietic development of induced pluripotent stem cells via Flk-1 $(+)$ hemoangiogenic progenitors. J Cell Physiol 2009; 221: 367.

52. Alexandre CS, Volpini RA, Shimizu MH, et al.: Lineage-negative bone marrow cells protect against chronic renal failure. Stem Cells 2009; 27: 682.

53. Semedo P, Palasio CG, Oliveira CD, et al:: Early modulation of inflammation by mesenchymal stem cell after acute kidney injury. Int Immunopharmacol 2009; 9: 677

54. Semedo P, Correa-Costa M, Antonio Cenedeze M et al:: Mesenchymal stem cells attenuate renal fibrosis through immune modulation and remodeling properties in a rat remnant kidney model. Stem Cells 2009; 27: 3063.

55. Nguyen BK, Maltais S, Perrault LP, et al:: Improved function and myocardial repair of infarcted heart by intracoronary injection of mesenchymal stem cell-derived growth factors. J Cardiovasc Transl Res 2010; 3: 547

56. Nemeth K, Keane-Myers A, Brown JM, et al.: Bone marrow stromal cells use TGF-beta to suppress allergic responses in a mouse model of ragweed-induced asthma. Proc Natl Acad Sci U S A 2010; 107: 5652

57. Li J, Zhu H, Liu Y, et al.: Human mesenchyma stem cell transplantation protects against cerebral ischemic injury and upregulates interleukin-10 expression in Macacafascicularis. Brain Res 2010 1334: 65

58. Wu Y, Chen L, Scott PG, and Tredget EE: Mesenchymal stem cells enhance wound healing 
through differentiation and angiogenesis. Stem Cells 2007; 25: 2648.

59. Sasaki M, Abe R, Fujita $Y$, Ando S, Inokuma D, and Shimizu H: Mesenchymal stem cells are recruited into wounded skin and contribute to wound repair by transdifferentiation into multiple skin cell type. J Immunol 2008; 180: 2581.

60. McLaughlin MM and Marra KG: The use of adipose-derived stem cells as sheets for wound healing. Organogenesis 2013; 9: 79.

61. Rouwkema J, Westerweel PE, de Boer J, Verhaar $M C$, and van Blitterswijk CA: The use of endothelial progenitor cells for prevascularized bone tissue engineering. Tissue Eng Part A 2009; 15: 2015.

62. Sukpat S, Isarasena N, Wongphoom J, and Patumraj S: Vasculoprotective effects of combined endothelial progenitor cells and mesenchymal stem cells in diabetic wound care: their potential role in decreasing wound-oxidative stress. BioMed Res Int 2013; 2013: 459196.

63. Keramaris NC, Kaptanis S, Moss HL, Loppini M, Pneumaticos S, and Maffulli N: Endothelial pro- genitor cells (EPCs) and mesenchymal stem cells (MSCs) in bone healing. Curr Stem Cell Res Ther 2012; 7: 293.

64. O'Loughlin A, Kulkarni M, Creane M, et al:: Topical administration of allogeneic mesenchymal stem cells seeded in a collagen scaffold augments wound healing and increases angiogenesis in the diabetic rabbit ulcer. Diabetes 2013; 62: 2588.

65. Takahashi K, Tanabe K, Ohnuki M, et al:: Induction of pluripotent stem cells from adult human fibroblasts by defined factors. Cell 2007; 131: 861 .

66. Yagi T, Kosakai A, Ito D, et al:: Establishment of induced pluripotent stem cells from centenarians for neurodegenerative disease research. PLoS One 2012; 7: e41572.

67. Zammaretti $\mathrm{P}$ and Zisch AH: Adult 'endothelial progenitor cells'. Renewing vasculature. Int $\mathrm{J}$ Biochem Cell Biol 2005; 37: 493.

68. Peng LH, Tsang SY, Tabata Y, and Gao JQ: Genetically-manipulated adult stem cells as therapeutic agents and gene delivery vehicle for wound repair and regeneration. J Control Release 2012; 157: 321.
69. Smadja DM, Cornet A, Emmerich J, Aiach M, and Gaussem P: Endothelial progenitor cells: characterization, in vitro expansion, and prospects for autologous cell therapy. Cell Biol Toxicol 2007; 23: 223.

\section{Abbreviations and Acronyms}

bFGF $=$ basic fibroblast growth factor CXCR-4 $=$ chemokine (C-X-C motif) receptor 4

$\mathrm{EPC}=$ endothelial progenitor cell

$\mathrm{ES}=$ embryonic stem cell GM-CSF = granulocyte-macrophage colony stimulating factor

HIF-1 $\alpha=$ hypoxia inducible factor-1 alpha

iPS cell $=$ induced-pluripotent stem cell

MMP-9 $=$ matrix metalloproteinase -9

$\mathrm{MNC}=$ mononuclear cell

$\mathrm{MSC}=$ mesenchymal stem cell

$\mathrm{SCF}=$ stem cell factor

SDF- $1=$ stromal cell derived factor- 1

$\mathrm{VEGF}=$ vascular endothelial growth factor 